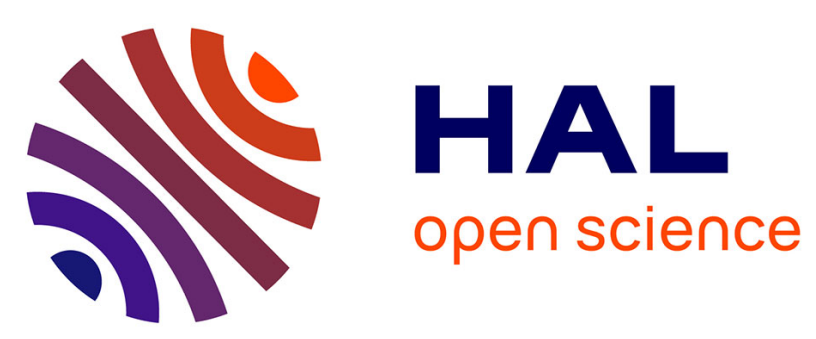

\title{
La Cognition entrepreneuriale. Enjeux et perspectives pour la recherche en entrepreneuriat
}

\author{
Christophe Schmitt, Denis A. Grégoire
}

\section{To cite this version:}

Christophe Schmitt, Denis A. Grégoire. La Cognition entrepreneuriale. Enjeux et perspectives pour la recherche en entrepreneuriat. Revue de l'Entrepreneuriat, 2019, 18 (1), pp.7-22. 10.3917/entre.181.0007 . hal-02365530

\section{HAL Id: hal-02365530 \\ https://hal.univ-lorraine.fr/hal-02365530}

Submitted on 7 Dec 2020

HAL is a multi-disciplinary open access archive for the deposit and dissemination of scientific research documents, whether they are published or not. The documents may come from teaching and research institutions in France or abroad, or from public or private research centers.
L'archive ouverte pluridisciplinaire HAL, est destinée au dépôt et à la diffusion de documents scientifiques de niveau recherche, publiés ou non, émanant des établissements d'enseignement et de recherche français ou étrangers, des laboratoires publics ou privés. 


\title{
LA COGNITION ENTREPRENEURIALE. ENJEUX ET PERSPECTIVES POUR LA RECHERCHE EN ENTREPRENEURIAT
}

\author{
Christophe Schmitt, Denis A. Grégoire
}

De Boeck Supérieur | « Revue de l’Entrepreneuriat »

2019/1 Vol. 18 | pages 7 à 22

ISSN 1766-2524

ISBN 9782807393066

Article disponible en ligne à l'adresse :

https://www.cairn.info/revue-de-l-entrepreneuriat-2019-1-page-7.htm

Distribution électronique Cairn.info pour De Boeck Supérieur.

(C) De Boeck Supérieur. Tous droits réservés pour tous pays.

La reproduction ou représentation de cet article, notamment par photocopie, n'est autorisée que dans les limites des conditions générales d'utilisation du site ou, le cas échéant, des conditions générales de la licence souscrite par votre établissement. Toute autre reproduction ou représentation, en tout ou partie, sous quelque forme et de quelque manière que ce soit, est interdite sauf accord préalable et écrit de l'éditeur, en dehors des cas prévus par la législation en vigueur en France. Il est précisé que son stockage dans une base de données est également interdit. 
$N^{\circ} 1$, vol. 18, 2019

\section{Éditorial}

\section{La cognition entrepreneuriale. Enjeux et perspectives pour la recherche en entrepreneuriat}

Entrepreneurial cognition.

Issues and perspectives for the study of entrepreneurship

Christophe Schmitt Titulaire de la Chaire Entreprendre IAE Metz/CEREFIGE - Université de Lorraine christophe.schmitt@univ-lorraine.fr

Denis A. Grégoire Chaire Rogers-J.A.-Bombardier de recherche en entrepreneuriat HEC Montréal 3000, Chemin de la Côte-Sainte-Catherine Montréal (Québec) CANADA H3T 2A7 denis.gregoire@hec.ca

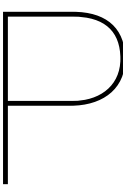

our bien situer la contribution des articles de ce numéro dans la trame des recherches contemporaines en entrepreneuriat, nous proposons un tour d'horizon des prémisses, notions et défis inhérents à la recherche sur les dynamiques affectives et cognitives de l'entrepreneuriat. Nous présentons ensuite les cinq articles du numéro thématique - soulignant au passage quelques éléments qui nous paraissent particulièrement riches d'enseignements. Nous concluons ce tour d'horizon par une brève synthèse des avenues de recherches qui nous semblent les plus prometteuses.

\section{- Mots clés : Cognition entrepreneuriale}




\begin{abstract}
-o help locate the contributions of this special issues' articles within contemporary studies of entrepreneurship, we propose a short overview of the premises, notions and challenges inherent to research on the affective and cognitive dynamics of entrepreneurship. We then present the five articles forming the special issue - highlighting in passing certain elements that seem particularly insightful. We conclude our overview with a brief summary of the most promising avenues of future research.
\end{abstract}

\title{
- Keywords : Entrepreneurial cognition
}

Ces dernières années, les travaux de recherche en entrepreneuriat ont de plus en plus attiré l'attention sur les dynamiques affectives et cognitives qui encouragent, supportent, voire contraignent les différentes dimensions de l'action entrepreneuriale (cf., Chabaud \& Sammut 2013 ; Cossette 2000 ; 2010 ; Fayolle \& Degeorge 2012 ; Filion 2008 ; Grégoire, Cornelissen, Dimov \& van Burg 2015 ; Schmitt 2015 ; 2017). Ces développements s'inscrivent en parallèle de plusieurs avancées cognitives dans les sciences du management (cf. Gavetti \& Ocasio 2016 ; Laroche \& Nioche 2006 ; 2015 ; Walsh 1995). En même temps, les recherches cognitives en entrepreneuriat ont connu leurs propres développements (cf. Baron 2004 ; Forbes 1999 ; Mitchell et al. 2002 ; 2004; 2007). Plus encore, ces recherches font face à des défis théoriques et méthodologiques spécifiques au phénomène de l'entrepreneuriat (voir Davidsson 2003 ; McMullen \& Dimov 2013 ; Shane \& Venkataraman 2000). Pour bien capturer l'essence du phénomène, les recherches en cognition entrepreneuriale doivent ainsi prendre en compte les hauts niveaux d'incertitude, de stress, d'engagement personnel, de charges émotives et d'autres contraintes internes et externes qui sont au cœur de l'action entrepreneuriale (Grégoire, D. A., Binder, J. K., \& Rauch, A. (2019). Navigating the validity tradeoffs of entrepreneurship research experiments: A systematic review and best-practice suggestions. Journal of Business Venturing, 34(2), 284-310.).

Une des caractéristiques de l'approche cognitive dans ce champ de recherche est d'envisager l'entrepreneuriat sous un angle différent de ce qu'avançaient les recherches passées sur les caractéristiques, la personnalité ou les motivations des entrepreneurs (cf. D'Andria \& Gabarret 2016 ; Dié 2016 ; Estaym Durrieu \& Diop, 2011 ; Séville \& Wirtz, 2010). II s'agit essentiellement " d'ouvrir la boîte noire » pour considérer la manière dont l'esprit humain envisage l'agir entrepreneurial. L'approche cognitive de l'entrepreneuriat incite à s'interroger sur la manière dont l'entrepreneur pense et agit, pour ainsi dépasser la seule influence de facteurs objectifs et autres contraintes externes à l'entrepreneur et mieux comprendre la dynamique de ses réflexions, de ses représentations, de son engagement, voire de ses émotions. L'approche cognitive apparaît ainsi comme un levier intéressant pour développer une meilleure compréhension de l'entrepreneuriat et de ses dynamiques.

Pour contribuer à avancer les recherches francophones mobilisant la perspective cognitive pour mieux comprendre de telles dynamiques, nous avons d'abord organisé une table ronde sur le sujet - discussions qui se sont tenues lors du $13^{e}$ Congrès International Francophone en Entrepreneuriat et PME (CIFEPME) qui eut lieu en octobre 2016 à I'Université du Québec à Trois-Rivières. C'est d'ailleurs en vue de cette table ronde qu'émergea l'idée de faire un numéro thématique sur le sujet dans la Revue de l'entrepreneuriat. Après plus de deux ans de préparation, d'évaluation de manuscrits et d'échanges avec les auteurs et évaluateurs, c'est donc avec grand plaisir - et beaucoup de fierté - que nous vous présentons 
$N^{\circ} 1$, vol. 18, 2019

ce numéro spécial de la Revue de l'entrepreneuriat portant sur la cognition entrepreneuriale. Les cinq articles formant ce numéro spécial sont le fruit du travail de 13 chercheurs œuvrant dans 10 institutions situées dans quatre pays différents, de part et d'autre de l'Atlantique. Qui plus est, la réalisation de ce numéro a bénéficié de l'appui indéfectible des deux rédacteurs en chef de la revue, les professeurs Didier Chabaud (IAE de Paris) et Sylvie Sammut (Université de Montpellier), sans compter la contribution essentielle de plus d'une trentaine d'évaluateurs. Nous remercions d'emblée toutes ces personnes pour leurs efforts. Le résultat offre une riche collection de pièces représentant ce qui se fait de mieux dans l'univers des recherches francophones sur la cognition entrepreneuriale.

Pour bien situer la contribution des cinq articles formant ce numéro spécial dans la trame des recherches contemporaines, nous proposons un tour d'horizon des prémisses, défis et enjeux caractérisant la recherche sur les dynamiques affectives et cognitives de l'entrepreneuriat. Nous présentons ensuite les cinq articles du numéro spécial - soulignant au passage quelques aspects de ces travaux qui nous apparaissent particulièrement riches d'enseignements. Nous concluons ce tour d'horizon par une brève synthèse des avenues de recherches qui nous semblent les plus prometteuses.

\section{Qu'est-ce que la cognition entrepreneuriale ?}

\subsection{Les origines et éléments constitutifs de la perspective cognitive}

D'un point de vue formel, les sciences cognitives se définissent comme l'étude de la pensée humaine et de ses processus (Andler 2004 ; Neisser 1967 ; Vignaux 1994). Au-delà de fondements dans des débats philosophiques comme la dichotomie corps-esprit (voir Descartes 2000/1637), dans les développements formels de la psychologie à partir du milieu du XIX siècle voire dans certaines avancées mathématiques durant la $2^{e}$ Guerre Mondiale, les sciences cognitives prirent véritablement leur essor au début des années 1950 - et plus particulièrement à travers la convergence d'un ensemble de développements parallèles dans les disciplines de l'anthropologie, de la linguistique, de la logique, des mathématiques, des neurosciences, de la philosophie, de la psychologie, de la psychologie et des sciences de l'informatique et de l'intelligence artificielle (cf. Gardner 1985 ; Miller 2003). En retour, les principales contributions des sciences cognitives portent sur des phénomènes comme l'acquisition et la mobilisation des connaissances, l'apprentissage, l'attention, la compréhension, la conscience, l'intelligence, l'interprétation, le langage, la mémoire, la perception, la prise de décision, le raisonnement, la résolution de problèmes, la signification et le symbolisme - et les implications de ces phénomènes sur les comportements humains (cf. Andler 2004 ; Frankish \& Ramsey 2012 ; Smith \& Osherson 1995). Par extension, les développements récents ont permis de transcender les oppositions traditionnelles entre cognition et émotion (voir Lazarus 1984 vs. Zajonc 1984), tant et si bien que certaines notions propres aux sciences cognitives peuvent parfois être utilisées dans l'étude de dynamiques affectives - et vice-versa (voir Gray 1990 ; Schwarz 2000). Au final, la cognition entrepreneuriale vient renforcer le paradigme de la décision (Schmitt 2017), c'est-à-dire comment les décisions sont prises (Hastie \& Dawes 2010).

En termes pratiques, la perspective cognitive est souvent expliquée à travers l'analogie avançant que l'esprit est au corps ce que le logiciel (software) est à l'ordinateur (hardware) (voir Broadbent 1958 ; Simon \& Newell 1964). De par cette analogie, l'objet des sciences cognitives consiste alors à décrire, expliquer et modéliser le ou les «logiciel(s) » cognitfis qui 
permettent aux structures physiologiques du cerveau (le " hardware ") de jouer un rôle dans la vaste gamme des comportements humains. Paul Thagard (1995 p. 10) résume cette idée à travers l'acronyme de Computational-Representational Understanding of the Mind (CRUM) la compréhension de l'esprit humain à travers un modèle computationnel et représentationnel. Cet acronyme reflète la prémisse qu'on peut modéliser la pensée humaine en faisant d'abord l'hypothèse de modèles mentaux structurés qui "représentent " l'information et les connaissances dans l'esprit humain (voir Johnson-Laird 2006), et ensuite de " procédures computationnelles » qui permettent de créer, emmagasiner, transformer et surtout, de mobiliser ces représentations mentales pour aider l'esprit humain à accomplir des tâches « raisonnées » (par exemple, apprendre à lire, compléter un puzzle, définir un plan de travail pour accomplir une tâche, faire un choix parmi différentes options, etc.).

Bien sûr, cette analogie fonctionnelle a été critiquée (e.g., Dellantonio \& Pastore 2017), en partie parce qu'elle tend à réduire la richesse de la pensée humaine à de simples mécanismes de perception et de traitement de l'information. En dépit de leur validité toutefois, ces critiques masquent la dimension intuitivement pédagogique de l'analogie : l'idée que les sciences cognitives cherchent à mieux comprendre les processus de la pensée humaine en développant à la fois des notions sur la représentation de l'information et des connaissances et sur les mécanismes mentaux qui mobilisent ces représentations dans la réalisation de diverses tâches propres à l'activité humaine.

\section{Les recherches d'inspiration cognitive en entrepreneuriat}

Synthétisant divers travaux sur les fondements et l'articulation des sciences cognitives et leur implication pour les recherches en entrepreneuriat, Grégoire, Corbett et McMullen (2011: p. 1445) ont attiré l'attention sur trois éléments constitutifs de l'approche cognitive:

1. Le mentalisme, c'est-à-dire une attention à étudier les représentations mentales que les individus se construisent d'eux-mêmes, des autres, de leurs circonstances, des contextes dans lesquels ils opèrent, ainsi que de leurs savoirs et autres états mentaux ;

2. Une orientation processuelle, à savoir un souci de modéliser les processus mentaux permettant l'acquisition, la formation, le stockage, la transformation, l'optimisation et l'utilisation de ces représentations mentales ; et

3. Un souci à considérer l'articulation de ces représentations et processus mentaux à travers plusieurs niveaux d'analyse - depuis les éléments constitutifs d'une représentation mentale ou son ancrage dans un système neuronal d'un individu jusqu'au partage de ces représentations au niveau de groupes d'individus, voire de populations plus larges.

En principe, ces éléments permettent de distinguer les recherches cognitives d'autres approches interprétatives ou d'inspiration subjective. Cela permet de préciser les frontières de ce qui relève ou non de la cognition entrepreneuriale. Ainsi, la perspective cognitive de l'entrepreneuriat ne se limite pas à simplement considérer le point de vue et les représentations des acteurs. Pour ancrer une recherche dans les sciences cognitives, il faut pouvoir expliquer quelque chose quant à la nature et/ou la structure des représentations mentales des individus, quant aux processus mentaux impliquant ces représentations et/ou quant à l'articulation de ces représentations et processus à travers différents niveaux d'analyse (voir Grégoire 2014).

Construisant sur ces bases, la cognition entrepreneuriale a pour intérêt de faire évoluer la recherche en entrepreneuriat où on passe de l'image de l'entrepreneur comme un simple 
$N^{\circ} 1$, vol. 18, 2019

processeur d'information à celle de processeur de représentations (Julien et al. 2003 ; Schmitt 2008). L'entrepreneuriat est envisagé non plus sous l'angle de l'information à optimiser, mais sous l'angle des représentations à organiser et à développer (Julien \& Schmitt 2008). Derrière cette interrogation, se profile une représentation de l'entrepreneuriat qui trouve ses racines dans la théorie des ressources stratégiques d'une part (Barney 1986) et la théorie du capital humain d'autre part (Becker 1976, Schultz 1961) et que l'on trouve à travers la théorie de l'effectuation (Sarasvathy 2001 ; 2003). Au moment où nous écrivons ces lignes, les recherches contemporaines sur la cognition entrepreneuriale dépassent largement le cadre plus strict des processus de perception et traitement de l'information et de l'analogie computationnelle. Tout comme les recherches d'inspiration cognitive dans les sciences du management, les recherches cognitives en entrepreneuriat mobilisent une large gamme de perspectives (Cossette 2000 ; 2010 ; Verstraete \& Fayolle 2005) allant de l'interprétativisme et du sensemaking (Gratton 2014 ; Lefebvre \& O'Shea 2013 ; Paradas 2007) jusqu'à la mobilisation de notions associées aux neurosciences ou à la cognition situés (Lièvre \& Rix-Lièvre 2011 ; Verzat 2013).

\section{Des débats en tension : l'apport de la cognition entrepreneuriale}

L'émergence de la perspective cognitive a contribué d'importances avancées dans les connaissances académiques en entrepreneuriat (Chabaud, Sammut \& Degeorge 2017 ; Grégoire et al. 2015). Par exemple, de telles avancées sont manifestes dans l'évolution des recherches sur l'intention entrepreneuriale - et sur les facteurs et dynamiques qui favorisent l'émergence et l'affirmation de telles intentions (cf. Alexandre-Leclair \& Redien-Collot 2013 ; Barbosa, De Oliveira, Fayolle \& Barbosa 2010 ; Delanoë \& Brulhart 2011 ; Yatribi, Balhadj \& Giraud 2017). Deux modèles ont particulièrement attiré l'attention : le modèle de Shapero (1975) et le modèle du comportement planifié d'Ajzen (1991). Bien que différents par leur contenu, ces deux modèles s'inscrivent dans une même logique de planification où les choix de l'entrepreneur sont considérés comme pensés et raisonnés (Schmitt, 2015). L'apport de la perspective cognitive dans ce champ d'étude a été proposé des leviers pour examiner comment la représentation que les individus se font des ressources et contraintes de leur environnement permet de mieux comprendre la formation de leurs intentions entrepreneuriales - voire la fluctuation de telles intentions. Concevoir les intentions entrepreneuriales comme un objet cognitif a aussi permis de jeter un œil renouvelé sur l'influence de dimensions affectives et identitaires dans la formation de telles intentions. Au final, la mobilisation de modèles issus de la cognition a permis un accroissement considérable de l'activité au sein de la communauté scientifique.

Les recherches sur les opportunités entrepreneuriales offrent un autre exemple de sujet où la perspective cognitive a permis d'importantes avancées. Initialement avancé comme central au champ de recherche par Venkataraman (1997 sur la base de Kirzner 1979 et autres), le concept d'opportunité a rapidement attiré l'attention de nombreux chercheurs - sans toutefois qu'il n'y ait de consensus autour de la définition du concept (cf. Davidsson 2015 ; Foss \& Klein 2018). Les premiers efforts d'articulation ont généralement avancé l'idée que les opportunités relèvent de circonstances antécédentes et indépendantes à l'action des entrepreneurs (e.g. Dutta \& Crossan 2005 ; Eckhardt \& Shane, 2003 ; Ngijol 2015). Conçues en ce sens, les opportunités en viennent à prendre une valeur quasi-objective. Le défi des entrepreneurs est alors de les reconnaître, pour ensuite créer le véhicule le mieux à même d'exploiter ces circonstances (i.e., produit, service, modèle d'affaires, entreprise, etc.). En contraste avec cette articulation, les recherches d'inspiration cognitive ont favorisé l'émergence d'articulations théoriques alternatives du concept d'opportunité. Sans pour autant trancher définitivement le débat sur la 
nature objective ou subjective opportunités, et en parallèle avec d'autres avancées (cf. Alvarez \& Barney 2007 ; Dimov 2007 ; Sarasvathy, 2001 ; 2008), les travaux sur la cognition entrepreneuriale sont venus renforcer l'idée que l'entrepreneur participe activement à la construction des opportunités (Cornelissen \& Clarke 2010 ; Grégoire, Barr \& Shepherd 2010 ; Mitchell et al. 2007 ; Navis \& Glynn 2010).

L'approche cognitive du concept d'opportunité implique que l'entrepreneur doit pouvoir se représenter une image de son environnement - et de l'action qu'il pourrait avoir sur cet environnement. Cela ouvre la porte à différentes études sur la manière dont les entrepreneurs conçoivent leurs idées - notamment par rapport à leurs savoirs et leurs expériences. Cela attire aussi l'attention sur les capacités des entrepreneurs à donner du sens à ces interactions environnement/savoirs/expérience pour créer des opportunités - offrant ainsi un écho pertinent aux travaux portant sur les signaux forts/faibles, à l'importance du capital social et du réseau et à l'articulation des opportunités dans le temps (Julien 2005 ; 2010 ; Warnier, Lecocq \& Demil 2018).

Toujours sur le même sujet, la perspective cognitive a aussi permis de jeter un regard sur les processus cognitifs influençant la génération d'idées d'innovation prometteuses - et sur l'influence de tels processus dans la perception de telles idées (voir Baron \& Ensley 2006 ; Degeorge \& Magakian 2013 ; Grégoire \& Shepherd 2012 ; Wood, McKelvie \& Haynie 2014). À travers tous ces développements, la cognition entrepreneuriale a joué un rôle de premier plan dans l'avancement des recherches sur les opportunités entrepreneuriales. De manière toute particulière, ces recherches ont permis de rompre avec la tradition de l'individualisme méthodologique pour proposer une approche plus en interaction avec l'environnement de l'entrepreneur. On peut ainsi s'attendre à ce que des recherches futures permettent de faire une synthèse de ces deux approches entre l'opportunité donnée et l'opportunité construite.

\section{L'émergence de nouveaux débats}

Les recherches contemporaines en entrepreneuriat en sont venues à mobiliser une large gamme de concepts, notions et méthodes propres aux sciences cognitives. Ces derniers temps toutefois, de nouveaux débats émergent au sein de la communauté scientifique. Ne pouvant être exhaustif, nous souhaitons faire écho à deux de ces débats. Le premier mobilise les concepts de cognition située et distribuée. Le second fait écho aux avancées théoriques et méthodologiques dans le domaine des neurosciences.

\subsection{De la cognition située à la cognition distribuée}

De manière sommaire, la notion de cognition située avance l'idée que la dimension rationnelle des comportements humains ne procède pas de seuls phénomènes mentaux intraindividuels, mais s'articule à travers les interactions dynamiques d'un système cognitive que l'agent forme avec son environnement (voir Laville 2000). Par extension, la notion de cognition distribuée étend ces dynamiques cognitives à l'échelle de groupes d'individus en interaction (ibidem). La cognition située trouve ses origines notamment dans les travaux des interactionnistes des années 1970, qui introduisent la dimension interprétative d'une action qui se fait en lien avec notamment avec d'autres individus à travers une situation donnée. Par ailleurs, l'introduction de la cognition située et de la cognition distribuée s'inscrivent clairement dans la remise en cause de l'individualisme méthodologique à travers lequel, la recherche en entrepreneuriat s'était construite, voire enfermée (Lièvre \& Rix-Lièvre 2011). Cette intégration de la cognition 
$N^{\circ} 1$, vol. 18, 2019

située puis de la cognition distribuée au sein de la recherche en entrepreneuriat se retrouve notamment à travers l'intersubjectivité mobilisée par Sarasvathy et Venkataraman (2011; mais voir aussi Dew, Grichnik, Mayer-Haug, Read \& Brinckmann 2015, Mitchell, Randolph-Seng \& Mitchell 2011 ; et Schmitt 2017).

L'intérêt de la cognition située en entrepreneuriat met l'accent principalement sur la dimension relationnelle de l'entrepreneur (Schmitt 2015 ; Verzat 2014). En mettant ainsi l'accent sur l'importance du réseau, la notion de cognition située vient interroger non seulement la dynamique des relations développées par l'entrepreneur mais aussi les évolutions de l'environnement de celui-ci. Cela renvoie à des recherches portant par exemple sur la construction des opportunités (Alvarez et Barney, 2007), l'improvisation (Julien \& Brunelle 2001) ou encore sur le bricolage (Jaouen \& Nakara 2015).

En ce qui concerne l'intérêt de la cognition distribuée, elle vient compléter la cognition située en proposant de prendre en considération la coopération entre l'entrepreneur et les différentes parties prenantes en lien avec l'entrepreneur (voir Toutain \& Salgado 2014). La cognition distribuée s'intéresse notamment à l'engagement de ces parties prenantes dans le processus entrepreneurial. Cela se traduit par exemple sur des recherches portant sur les équipes entrepreneuriales et leurs dynamiques (Ben-Hafaïdeh 2006), la gouvernance en lien avec les business angel (Bessière et Stéphany 2015).

Ces deux orientations montrent non seulement le dynamisme actuel au niveau de la recherche en entrepreneuriat mais aussi la volonté de sophistication conceptuelle du champ pour améliorer la compréhension de l'entrepreneuriat en développant de nouvelles connaissances. Toutefois, il ne faut pas perdre de vue la nécessité d'opérationnaliser ces concepts afin de ne pas rester à un niveau purement conceptuel. Dans cette optique, il nous semble important d'encourager les chercheurs à aller au-delà de la publication d'articles conceptuels pour plutôt s'attaquer à l'articulation méthodologique de ce que peuvent impliquer les perspectives de cognition située et distribuée pour l'entrepreneuriat. Cela demandera sûrement des efforts importants - mais nous croyons que le jeu en vaut la chandelle.

\subsection{Le potentiel des neurosciences}

En parallèle avec l'intérêt grandissant pour la cognition située et distribuée, plusieurs études récentes ont cherché à mobiliser davantage les avancées scientifiques contemporaines ayant porté sur les dynamiques biologiques et physiologiques des comportements humains. Par exemple, un certain nombre de chercheurs ont commencé à explorer les liens entre l'agir entrepreneurial et les mécanismes physiologiques expliquant les désordres mentaux (e.g., Guiliani \& Torrès 2017 ; Lerner, Hatak \& Rauch 2018 ; Wiklund, Hatak, Patzelt \& Shepherd 2018). De manière plus générale, quelques études ont cherché à tirer parti des développements théoriques et méthodologiques propres aux neurosciences (cf. Brentzen 2018 ; Krueger \& Welpe 2014 ; Nicolaou \& Shane 2014 ; Tracey \& Schluppeck 2014). Par exemple, quelques études ont mobilisé des techniques d'imagerie neuronales (voir Jacquemin \& Janssen 2013 ; Ward, Reeck \& Becker 2017) pour examiner quelles structures physiologiques du cerveau étaient mobilisées pour différentes tâches ou activités entrepreneuriales (e.g., Drover, Cerg \& Shane 2016 ; Laureiro-Martínez, Brusoni, Canessa \& Zollo 2015).

Ces recherches ouvrent de nouvelles frontières. En même temps, l'ajout d'observations descriptives des bases neurophysiologiques des comportements entrepreneuriaux n'amène pas nécessairement une meilleure compréhension des dynamiques affectives et/ou cognitives 
de l'entrepreneuriat. Pour véritablement exploiter le potentiel des neurosciences, il importe de pouvoir ajouter quelque chose d'inédit et/ou de plus profond aux théories et explications existantes. En ce sens, l'apport des neurosciences en entrepreneuriat reste encore un projet en devenir. Le défi reste d'aller au-delà des simples observations descriptives pour proposer de meilleures explications.

\section{Contributions des articles dans le numéro}

Ce numéro thématique de la Revue de l'entrepreneuriat met en scène cinq articles traitant de thèmes aussi variés que la théorie des focus régulateurs, l'innovation responsable dans les PME, les mécanismes de décision dans la reprise d'entreprise, l'apport de méthodes basées sur les cartes conceptuelles ou encore les postulats tacites en éducation entrepreneuriale. Chacun à leur façon, ces articles offrent différentes pistes de réflexion pour avancer une meilleure compréhension des dynamiques de l'entrepreneuriat.

Dans le premier article du numéro, Vincent Angel ( $U$. de Bordeaux) et Julie Hermans (UCLouvain) proposent une série de recommandations pour augmenter l'apport des recherches en entrepreneuriat mobilisant la théorie des focus régulateurs (TFR) développée par Higgins (1997 ; 1998). Après une synthèse des principales notions au cœur de cette théorie, Angel et Hermans offrent une analyse critique de quelque 33 articles pertinents ayant mobilisé la TFR en entrepreneuriat. Fort de ce tour d'horizon exhaustif des recherches passées mais aussi, de leur compréhension unique des enjeux théoriques et méthodologiques propres à l'application de cette théorie au phénomène de l'entrepreneuriat, Angel et Hermans identifient trois axes d'amélioration pour lesquelles ils proposent neuf recommandations. Ce faisant, Angel et Hermans offrent un article particulièrement riche d'enseignements pour aider à redynamiser les recherches sur la motivation des entrepreneurs. De manière plus spécifique, ces auteurs offrent de nouvelles avenues pour comprendre les dynamiques affectives et cognitives par lesquelles les entrepreneurs parviennent à moduler l'intensité de leurs efforts. Par ailleurs, le texte d'Angel et Hermans se démarque par ses réels efforts à encourager l'étude des focus régulateurs audelà des seules dynamiques intra-individuelles. Ils contribuent ainsi à articuler les recherches en cognition entrepreneuriale au niveau des dynamiques entre les membres d'une équipe, voire au niveau de l'organisation comme telle - faisant ainsi écho aux notions de cognition distribuée.

Dans le second article, Martin Cloutier (ESG UQAM), Sandrine Cueille (Université de Pau et des Pays de l'Adour), Gilles Recasens (Université de Pau et des Pays de l'Adour) et Scott Rosas (Concept Systems Inc) s'intéressent aux représentations des acteurs en lien avec l'entrepreneuriat. Ils nous proposent une approche collective et ascendante de la cognition entrepreneuriale autour de la notion de croissance des TPE. L'originalité proposée dans cet article, du point de vue cognitif, relève de la confrontation des représentations des entrepreneurs et des accompagnateurs - représentations qui peuvent s'avérer très différentes entre les uns et les autres. L'intérêt de l'article repose aussi sur le positionnement épistémo-méthodologique proposé par les auteurs autour du réalisme critique. Au final, les convergences et les divergences de représentations apparaissent comme des leviers pour l'action entrepreneuriale.

Pour sa part, le troisième article mobilise la perspective cognitive pour ni plus ni moins que questionner les vertus de l'éducation entrepreneuriale. Puisant sur le concept d'imaginaire social avancé par Castoriadis (1975) et sur les travaux de Bertrand (2015) sur les différentes théories et philosophies de l'éducation, Fabienne Bornard (INSEEC Business School), Caroline Verzat (ESCP Europe) et Chrystelle Gaujard (YNCREA) examinent les représentations 
$N^{\circ} 1$, vol. 18, 2019

cognitives partagées par différents acteurs de l'éducation entrepreneuriale. Dans une première étude qualitative, une série d'ateliers créatifs avec différents acteurs de l'éducation entrepreneuriale a permis de dégager une typologie de quatre représentations de l'éducation entrepreneuriale. Dans une seconde étude quantitative, les auteurs ont examiné la désirabilité de ces représentations. Ce faisant, les auteurs révèlent que derrière le large consensus superficiel entourant l'éducation entrepreneuriale se cachent de très grandes divergences quant aux finalités et pratiques éducatives de l'entrepreneuriat. Au-delà de son point de départ polémique, l'intérêt particulier de l'article de Bornard, Verzat et Gaujard est d'abord de montrer comment l'existence parallèle de différentes représentations de l'éducation entrepreneuriale induit d'importantes tensions entre les dimensions économiques et sociales de l'entrepreneuriat. En retour, les auteures proposent de résoudre ces tensions en fondant l'action de l'éducateur contemporain sur la posture pragmatiste de Dewey à savoir, de " fonder l'éducation sur la pensée systématique de l'expérience (...) ; le rôle de l'éducateur devient alors d'aider les jeunes à problématiser, à articuler de manière dialectique doute et certitude, à mener un processus d'enquête à base de questionnement. "

Le quatrième article s'intéresse tout particulièrement au lien entre innovation entrepreneuriale et Responsabilité Sociale de l'Entreprise (RSE) au sein d'une PME. Mantiaba CoulibalyBallet (Université Côte d'Azur) et Djamila Elidrissi (Université Côte d'Azur) propose de s'intéresser à ce lien sous l'angle de la cognition entrepreneuriale. Les auteurs mobilisent une méthodologique qualitative afin de comprendre les mécanismes cognitifs des cadres intermédiaires en situation. Elles montrent notamment que l'innovation entrepreneuriale résulte des mécanismes cognitifs individuels et collectifs entre la direction et le gestionnaire de niveau intermédiaire. Le lien entre cognition individuelle et collective ressort comment un enjeu essentiel dans les projets d'innovation de PME, faisant ainsi écho aux notions de cognition située évoquée précédemment.

Dans le cinquième et dernier article, Karim Mouhli (Université de Bretagne Occidentale) et Robert Paturel (Université de Bretagne Occidentale) nous offrent un angle original pour aborder la cognition entrepreneuriale : la reprise d'entreprise. Les auteurs s'intéressent notamment à l'importance de la construction de sens autour du processus de reprise d'entreprise. Cette logique se retrouve particulièrement au niveau des repreneurs consécutivement à une rupture professionnelle. À partir d'une étude exploratoire portant sur trois études de cas, les auteurs montrent l'importance de l'intersubjectivité dans la situation spécifique mettant en relation le cédant et le futur repreneur. L'article se termine par une proposition de modélisation du processus de reprise d'entreprise prenant en considération des facteurs d'influence contextuels comme l'histoire des personnes ou encore l'environnement social. Cet article raisonne aussi comme un hommage posthume de l'apport de Robert Paturel au champ de l'entrepreneuriat en général et de la reprise d'entreprise en particulier.

\section{Avenues de dépassement}

Arrivé au bout de ce bref tour d'horizon, nous ne pouvons que nous réjouir des contributions de ces cinq équipes de chercheurs. À n'en point douter, les recherches francophones sur les dynamiques cognitives supportant l'agir entrepreneurial se développent bien - et elles promettent de beaux lendemains ! C'est donc avec beaucoup d'anticipation que nous attendons de lire et/ou d'entendre les futurs travaux qu'inspireront les cinq articles de ce numéro thématique. 
Pour faciliter de telles avancées, il nous semble utile de conclure en réitérant ici quelques-uns des encouragements évoqués plus haut quant à l'utilité de dépasser la seule publication d'articles conceptuels. Pour bien exploiter tout le potentiel qu'offrent les sciences cognitives, il nous semble important d'encourager les chercheurs à tester leurs développements conceptuels "sur le terrain ». Dès les premiers balbutiements formels des années 1950 (voir Gardner 1985), le développement des sciences cognitives s'est accompagné d'importantes avancées méthodologiques pour essayer de mieux « capter » les représentations mentales des individus - et la mobilisation de ces représentations par l'esprit humain. Parmi plusieurs exemples pertinents, on note l'usage de simulations informatiques (e.g., Anderson 1996 ; Gavetti \& Levinthal 2000) mais aussi de techniques comme les cartes cognitives (Cossette 1996 ; Verstraete 1997) ou l'analyse de protocoles verbaux (Ericsson \& Simon 1993 ; Grégoire, Barr \& Shepherd 2010 ; Sarasvathy 2008).

Construisant sur de telles avancées méthodologiques, plusieurs travaux récents ont misé sur l'émergence de nouvelles technologies de collecte de données pour avancer une meilleure compréhension des dynamiques cognitives de l'entrepreneuriat. Par exemple, Foo, Uy et leurs collègues ont mobilisé la méthode de l'échantillonnage des expériences - et l'utilisation de micro sondages déployés à travers des services de messagerie SMS - pour examiner les liens entre émotions ressenties et efforts chez divers groupes d'entrepreneurs (cf. Foo, Uy \& Baron 2009 ; Uy, Foo \& Aguinis 2010). D'autres ont commencé à tirer parti de techniques d'imagerie neuronales (voir Jacquemin \& Janssen 2013 ; Ward, Reeck \& Becker 2017) pour examiner le rôle de différentes structures physiologiques du cerveau dans la réalisation de différentes tâches cognitives propres à l'entrepreneuriat. De notre point de vue, les défis les plus difficiles à surmonter pour avancer les recherches cognitives en entrepreneuriat on moins à voir avec la maîtrise des nouvelles technologies de mesure (voir Scherbaum \& Meade 2013), mais concernent l'articulation de designs de recherche et de stratégies méthodologiques logiquement cohérents avec les développements théoriques avancés (voir Grégoire 2014 ; Grégoire \& Schurer-Lambert 2014). Dans cette optique, nous espérons que la publication de ce numéro thématique favorisera l'émergence d'une communauté de chercheurs francophones intéressés à discuter de leurs projets, difficultés et ambitions.

\section{Bibliographie}

Alexandre-Leclair, L., \& Redien-Collot, R. (2013). L'intention entrepreneuriale des femmes : le cas de l'Égypte. Revue Internationale PME, 26(1), 93-116.

Alvarez, S.A. \& Barney, J.B. (2007). Discovery and creation : alternative theories of entrepreneurial creation. Strategic Entrepreneurship Journal, 1(1), 11-26.

Andler, D. (Dir.) (2004). Introduction aux sciences cognitives. Paris, FR : Folio Essais.

Ajzen, I. (1991). The theory of planned behavior. Organizational Behavior and Human Decision Processes, 50(2), 179-211.

Anderson, J. R. (1996). ACT: A simple theory of complex cognition. American Psychologist, 51(4), 355.

Barbosa, S.D., De Oliveira, W.M., Fayolle, A., \& Barbosa, F.V. (2010). Perceptions culturelles et intention d'entreprendre Une comparaison entre des étudiants brésiliens et français. Revue Internationale PME, 23(2).

Barney, J.B. (1986). Strategic Factor Markets: Expectations, Luck, and Business Strategy, Management Science, 32(10): 1231-1241. 
$N^{\circ} 1$, vol. 18, 2019

Baron, R.A. (2004). The cognitive perspective: A valuable tool for answering entrepreneurship's basic "why" questions. Journal of Business Venturing, 19, 221-39.

Baron, R.A. \& Ensley, M.D. (2006). Opportunity recognition as the detection of meaningful patterns: evidence from comparisons of novice and experienced entrepreneurs. Management Science, 52(9), 1331-1344.

Becker G. (1976). Crime and Punishment: An Economic Approach, Journal of Political Economy, vol. $76, N^{\circ} 2$, p. $170-195$.

Ben-Hafaïedh, C. (2006). Entrepreneuriat en équipe: positionnement dans le champ de l'entrepreneuriat collectif. Revue de l'Entrepreneuriat, 5(2), 31-54.

Bentzen, J.S. (2018). Cognitive neuroscience perspective on entrepreneurship. In R.V. Turcan \& N.M. Fraser (Eds.), The Palgrave Handbook of Multidisciplinary Perspectives on Entrepreneurship, pp. 43-58. London, UK: Palgrave Macmillan.

Bertrand, Y. (2015). Les philosophies contemporaines de l'éducation. Québec, Canada : Faubert.

Bessière, V. \& Stéphany, E. (2015). Financement et gouvernance des start-ups en equitycrowdfunding, Finance Contrôle Stratégie [En ligne], 18-4 |

Broadbent, D.E. (1958). Perception and Communication. New York, NY: Pergamon.

Brockner, J., Higgins, E.R. \& Low, M.B. (2004). Regulatory focus theory and the entrepreneurial process. Journal of Business Venturing, 19(2), 203-220.

Bryant, P. (2007). Self-regulation and decision heuristics in entrepreneurial opportunity evaluation and exploitation. Management Decision, 45(4), 732-748.

Castoriadis, C. (1975). L'institution imaginaire de la société. Paris, FR : Seuil.

Chabaud D. et Sammut S. (2013). Éditorial: Entrepreneuriat et cognition. Revue de l'Entrepreneuriat, 4(12), 7-9.

Chabaud, D., Sammut, S., \& Degeorge, J.M. (2017). De l'intention à l'action entrepreneuriale: antécédents, écarts et chainons manquants. Revue de l'Entrepreneuriat, 16(3), 7-15.

Cornelissen, J.P. \& Clarke, J.S. (2010). Imagining and rationalizing opportunities: inductive reasoning and the creation and justification of new ventures. Academy of Management Review, 35(4), 539-557.

Cossette, P. (1996). La vision stratégique du propriétaire-dirigeant de PME: étude de cartographie cognitive. Revue Internationale PME, 9(1), 123-142.

Cossette, P. (2000). La cognition comme objet d'étude dans la littérature scientifique sur la PME et l'entrepreneuriat. Revue Internationale PME, 13(1), 11-37.

Cossette, P. (2010). Usages du concept de cognition dans les travaux de recherche sur l'entrepreneuriat. Revue Internationale PME, 23(1), 9-32.

d'Andria, A., \& Gabarret, I. (2016). Femmes et entrepreneurs: trente ans de recherches en motivation entrepreneuriale féminine. Revue de l'Entrepreneuriat, 15(3), 87-107.

Davidsson, P. (2003). The domain of entrepreneurship research: Some suggestions. In J.A. Katz \& D.A. Shepherd (Editors), Cognitive Approaches to Entrepreneurship Research, pp. 315-372). Oxford, UK: Emerald Group Publishing Limited.

Davidsson, P. (2015). Entrepreneurial opportunities and the entrepreneurship nexus: A re-conceptualization. Journal of Business Venturing, 30(5), 674-695.

Davidsson, P. (2016). A "business researcher" view on opportunities for psychology in entrepreneurship research. Applied Psychology, 65(3), 628-636. 
Degeorge, J.M., \& Magakian, J.L. (2013). Vision et opportunité entrepreneuriale: une relation au travers du processus d'idéation?. Gestion 2000, 30(4), 123-140.

Delanoë, S., \& Brulhart, F. (2011). Analyse des antécédents de l'intention entrepreneuriale de porteurs de projets français1. Revue Internationale PME, 24(1), 43-70.

Dellantonio, S. \& Pastore, L. (2017). The misleading aspects of the mind/computer analogy. In. S. Derllantonio \& L. Pastore (Eds.), Internal Perception: The Role of Bodily Information in Concepts and Word Mastery, pp. 47-97. Berlin, D: Springer.

Descartes, R. (2000/1637). Discours de la méthode. Paris, FR: Fayard / Mille et une nuits.

Dew, N., Grichnik, D., Mayer-Haug, K., Read, S., \& Brinckmann, J. (2015). Situated entrepreneurial cognition. International Journal of Management Reviews, 17(2), 143-164.

Dié, M. D. (2016). Caractéristiques de l'entrepreneur et défaillance des entreprises de la filière du livre au Cameroun. Revue de l'Entrepreneuriat, 15(3), 283-309.

Dimov, D. (2007). From opportunity insight to opportunity intention: the importance of person-situation learning match. Enterpreneurship Theory and Practice, 31(4), 561-583.

Drover, W., Cerf, M., \& Shane, S. (2016). The neuroscience of investor decision making: An fMRI study. Frontiers of Entrepreneurship Research, 36(1), 21.

Dutta, D.K. \& Crossan, M.M. (2005). The nature of entrepreneurial opportunities: understanding the process using the $4 \mathrm{l}$ organizational learning framework. Entrepreneurship Theory and Practice, 29(4), 425-449.

Eckhardt, J.R. \& Shane S.A. (2003. Opportunities and entrepreneurship. Journal of Management, 29(3), 333-349.

Ericsson, K. A., \& Simon, H. A. (1993). Protocol analysis. Cambridge, MA: MIT press.

Estay, C., Durrieu, F., \& Diop, P. (2011). Motivation entrepreneuriale et logique d'action du créateur. Revue Internationale PME, 24(1), 135-165.

Fayolle, A. et Degeorge, J.-M. (2012). Dynamique entrepreneuriale, le comportement de l'entrepreneur. Bruxelles, BE: De Boeck.

Filion, L.-J. (2008). Les représentations entrepreneuriales : un champ d'études en émergence. Revue Internationale de Psychosociologie, v14(32), p. 13-43.

Foo, M.D., Uy, M.A., \& Baron, R.A. (2009). How do feelings influence effort? An empirical study of entrepreneurs' affect and venture effort. Journal of Applied Psychology, 94(4), 1086.

Forbes, D.P. (1999). Cognitive approaches to new venture creation. International Journal of Management Reviews, 1, 415-39.

Foss, N. \& Klein, P. (2018). Entrepreneurial opportunities: Who needs them? Academy of Management Perspectives, https://doi.org/10.5465/amp.2017.0181

Frankish, K. \& Ramsey, W.M. (Eds). (2012). The Cambridge Handbook of Cognitive Science. Cambridge, UK : Cambridge University Press.

Gardner, H. (1985). The Mind's New Science: A History of the Cognitive Tevolution. New York, NY: BasicBooks.

Gavetti, G., \& Levinthal, D. (2000). Looking forward and looking backward: Cognitive and experiential search. Administrative Science Quarterly, 45(1), 113-137.

Gavetti, G. \& Ocasio, W. (2016). Introduction. In G. Gavetti \& W. Ocasio (Eds.), Advances in Strategic Management 32 (Cognition and Strategy). Bingley, UK: Emerald. 
$N^{\circ} 1$, vol. 18, 2019

Gratton, P. (2014). Pilotage de la pérennité organisationnelle: normes, représentations et contrôle. Revue Internationale PME, 27(3-4).

Gray, J.A. (1990). Brain systems that mediate both emotion and cognition. Cognition \& Emotion, 4(3), 269-288.

Grégoire, D.A. 2014. Exploring the affective and cognitive dynamics of entrepreneurship across time and planes of influence. In J.R. Mitchell, R.K. Mitchell \& B. Randolph-Seng (Editors): Handbook of Entrepreneurial Cognition, pp. 182-226. Northampton, MA: Edward Elgar.

Grégoire, D. A., Barr, P. S., \& Shepherd, D. A. (2010). Cognitive processes of opportunity recognition: The role of structural alignment. Organization Science, 21(2), 413-431.

Grégoire, D. A., Binder, J. K., \& Rauch, A. (2019). Navigating the validity tradeoffs of entrepreneurship research experiments: A systemaatic review and best-practice suggestions. Journal of Business Venturing, 34(2), 284-310.

Grégoire, D.A., Corbett, A.C. et McMullen, J.S. (2011). The cognitive perspective in entrepreneurship: An agenda for future research, Journal of Management Studies, 48(6), 1443-1477.

Grégoire, D.A., Cornelissen, J., Dimov, D., \& van Burg, E. (2015). The mind in the middle: Taking stock of affect and cognition research in entrepreneurship. International Journal of Management Reviews, 17(2): 125-142.

Grégoire, D.A. \& Schurer-Lambert, L. (2014). Getting inside entrepreneurs' heart and mind: Methods for advancing entrepreneurship research on affect and cognition. In E. Baker \& F. Welter (Editors), Routledge Companion to Entrepreneurship, pp. 450-466. Milton Park, Abingdon: UK: Routledge.

Grégoire, D. A., \& Shepherd, D. A. (2012). Technology-market combinations and the identification of entrepreneurial opportunities: An investigation of the opportunity-individual nexus. Academy of Management Journal, 55(4), 753-785.

Guiliani, F., \& Torrès, O. (2017). L'influence de la somnolence et de la concentration sur la vigilance entrepreneuriale des dirigeants de PME. Revue de l'Entrepreneuriat, 16(3), 147-176.

Hastie, R. \& Dawes, R.M. (2010). Rational choice in an uncertain world : The psychology of judgment and decision making. Thousand Oaks, CA : Sage.

Higgins, E.T. (1997). Beyond pleasure and pain. American Psychologist, 52(12), 1280-1300

Higgins, E.T. (1998). Promotion and prevention: Regulatory focus as a motivational principle, Advances in Experimental Social Psychology, 30, 1-46.

Jacquemin, A., \& Janssen, F. (2013). La cognition de l'entrepreneur: recherche interdisciplinaire et pratiques d'enseignement. Une interview du Professeur Norris F. KRUEGER, Jr. Revue de l'Entrepreneuriat, 12(1), 171-177.

Jaouen, A., Nakara, W.A. (2015). 'Bricolage' in the Implementation and the Use of IS by Micro-firms: An Empirical Study. In Rocha A., Correia A.M., Costanzo S. \& Reis L.P. (Eds), New Contributions in Information Systems and Technologies. Springer International Publishing, Switzerland, 449-458

Johnson-Laird, P. (2006). How We Reason. Oxford, UK: Oxford University Press.

Julien, P.-A. Brunelle, L. (2001). The gazelles, the jazz band metaphor and further: a case study, Communication présentée à la $2^{\text {nd }}$ international conference on dynamic enterprises. Portorož, Ljubljana, Slovénie. 
Julien, P.-A., Raymond L., Jacob, R. et Abdul-Nour, G. (2003). L'entreprise-réseau : concepts et applications, dix ans d'expérience de la chaire bombardier produits récréatifs - 1993-2003, Québec, Presses de l'université du Québec.

Julien, P.-A. (2005). Entrepreneuriat régional et économie de la connaissance. Québec, Presses universitaire du Québec.

Julien, P. A. (2010). Opportunités, information et temps. Revue de l'Entrepreneuriat, 9(1), 29-49.

Julien, P.-A. et Schmitt, C. (2008). Pour une vision renouvelée des pratiques entrepreneuriales, de la vision libérale à la vision sociale de l'entrepreneuriat, dans Schmitt, C. (dir) (2008) Regards sur l'évolution des pratiques entrepreneuriales, Québec, Presses de l'université du Québec, p. 15-34.

Kirzner, I.M. (1979). Perception, Opportunity and Profit. Chicago: University of Chicago Press.

Krueger, N., \& Welpe, I. (2014). Neuroentrepreneurship: what can entrepreneurship learn from neuroscience. Annals of Entrepreneurship Education and Pedagogy, 60-90.

Laroche, H. \& Nioche, J. (2006). L'approche cognitive de la stratégie d'entreprise. Revue française de gestion, 160(1), 81-105.

Laroche, H., \& Nioche, J. P. (2015). L'approche cognitive de la stratégie d'entreprise. Revue française de gestion, 41(253), 97-120.

Laureiro-Martínez, D., Brusoni, S., Canessa, N., \& Zollo, M. (2015). Understanding the explorationexploitation dilemma: An fMRI study of attention control and decision-making performance. Strategic Management Journal, 36(3), 319-338.

Laville, F. (2000). La cognition située: une nouvelle approche de la rationalité limitée. Revue Économique, 1301-1331.

Lazarus, R. (1984). On the primacy of cognition. American Psychologist, 39(2), 124-129.

Lefebvre, M., \& O'Shea, N. (2013). Intuition et succès entrepreneurial: La représentation sociale de l'intuition dans la presse française. Revue Internationale PME, 26(3-4), 169-186.

Lerner, D.A., Hatak, I., \& Rauch, A. (2018). Deep roots? Behavioral inhibition and behavioral activation system (BIS/BAS) sensitivity and entrepreneurship. Journal of Business Venturing Insights, 9 , 107-115.

Lièvre, P., \& Rix-Lièvre, G. (2011). Proposition d'un design de recherche pour investir le projet entrepreneurial: la question méthodologique de l'investigation de l'activité en situation. Revue de l'Entrepreneuriat, 10(2), 69-88.

McMullen, J. S., \& Dimov, D. (2013). Time and the entrepreneurial journey: The problems and promise of studying entrepreneurship as a process. Journal of Management Studies, 50(8), 1481-1512.

Miller, G.A. (2003). The cognitive revolution: A historical perspective. Trends in Cognitive Sciences, 7(3), 141-144.

Mitchell, R.K., Busenitz, L., Lant, T., McDougall, P.P., Morse, E.A., \& Smith, J.B. (2002). Toward a theory of entrepreneurial cognition: Rethinking the people side of entrepreneurship research. Entrepreneurship Theory and Practice, 27(2), 93-104.

Mitchell, R.K., Busenitz, L., Lant, T., McDougall, P.P., Morse, E. A., \& Smith, J.B. (2004). The distinctive and inclusive domain of entrepreneurial cognition research. Entrepreneurship Theory and Practice, 28(6), 505-518.

Mitchell, R.K., Busenitz, L.W., Bird, B., Marie Gaglio, C., McMullen, J.S., Morse, E.A., \& Smith, J.B. (2007). The central question in entrepreneurial cognition research 2007. Entrepreneurship Theory and Practice, 31(1), 1-27. 
$N^{\circ} 1$, vol. 18, 2019

Mitchell, R.K., Randolph-Seng, B. and Mitchell, J.R. 2011. Socially situated cognition: Imagining new opportunities for entrepreneurship research (Dialogue). Academy of Management Review, 36(4): 774-8.

Navis, C. \& Glynn, M.A. (2010). How new market categories emerge: temporal dynamics of legitimacy, identity, and entrepreneurship in satellite radio, 1990-2005. Administrative Science Quarterly, 55(3), 439-471.

Neisser, U. (1967). Cognitive Psychology. New York, NY: Appleton-Century-Crofts.

Nicolaou, N. \& Shane, S. (2014). Biology, neuroscience, and entrepreneurship. Journal of Management Inquiry, 23(1): 98-100.

Ngijol, J. (2015). Israel M. Kirzner: les opportunités au cœur de la dynamique entrepreneuriale. Revue de l'Entrepreneuriat, 14(4), 99-115.

Paradas, A. (2007). Le dirigeant comme levier de la RSE en TPE: approche exploratoire basée sur l'utilisation de récits et d'une cartographie cognitive. Revue Internationale PME, 20(3-4), 43-67.

Sarasvathy, S.D. 2001. Causation and effectuation: Toward a theoretical shift from economic inevitability to entrepreneurial contingency. Academy of Management Review, 26(2), 243-288.

Sarasvathy, S.D. 2003. Entrepreneurship as a science of the artificial. Journal of Economic Psychology, 24(2), 203-220.

Sarasvathy, S.D. 2008. Effectuation: Elements of Entrepreneurial Expertise. Northampton, MA: Edward Elgar Publishing.

Sarasvathy, S. Venkataraman, S. (2011). Entrepreneurship as method: open questions for an entrepreneurial future. Entrepreneurship Theory and Practice, Volume: 35 issue: 1, page(s): 113-135.

Scherbaum, C.A., \& Meade, A.W. (2013). New directions for measurement in management research. International Journal of Management Reviews, 15(2), 132-148.

Schmitt, C. (2008), Regards sur l'évolution des pratiques entrepreneuriales, Presses de l'Université du Québec.

Schmitt, C. (2015). L'agir entrepreneurial: Repenser l'action des entrepreneurs. Montréal, Québec : Presses de l'Université du Québec.

Schmitt, C. (2017). La fabrique de l'entrepreneuriat, Dunod, Paris.

Schultz, T.W. (1961). Investment in Human Capital, American Economic Review, Vol.51, p. 1-17

Schwarz, N. (2000). Emotion, cognition, and decision making. Cognition \& Emotion, 14(4), 433-440.

Séville, M., \& Wirtz, P. (2010). Caractéristiques et dynamique de l'équipe dirigeante dans une jeune entreprise en hypercroissance. Revue internationale PME, 23(3-4), 43-70.

Shapero, A. (1975). The Displaced, Uncomfortable Entrepreneur. Psychology Today, Vol. 9, Issue 6, p. 83-88.

Shane, S. \& Venkataraman, S. (2000). The promise of entrepreneurship as a field of research. Academy of Management Review, 25(1), 217-226.

Simon, H.A. and Newell, A. (1964). Information processing in computer and man. American Scientist, 52(3): 281-300.

Smith, E.E. \& Osherson, D.N. (Eds) (1995). Thinking. Volume 3 in An Invitation to Cognitive Science. Cambridge, MA : MIT Press.

Thagard, P. (2005). Mind: Introduction to Cognitive Science. Cambridge, MA: MIT Press. 
Toutain, O., \& Salgado, M. (2014). Quels sont les effets des pédagogies actives dans l'apprentissage de l'entrepreneuriat?. Revue de l'Entrepreneuriat, 13(2), 55-88.

Tracey, P., \& Schluppeck, D. (2014). Neuroentreprenuership: "Brain Pornography" or New Frontier in Entrepreneurship Research? Journal of Management Inquiry, 23(1): 101-103.

Uy, M.A., Foo, M.D. \& Aguinis, H. (2010). Using experience sampling methodology to advance entrepreneurship theory and research. Organizational Research Methods, 13(1), 31-54.

Venkataraman, S. (1997). The distinctive domain of entrepreneurship research. In Katz J. et Brockhaus R. (Eds.), Advances in Entrepreneurship, Firm Emergence, and Growth, pp. 119-138. Greenwich, CT: JAI Pres.

Verstraete, T. (1997). Cartographie cognitive et accompagnement du créateur d'entreprise. Revue Internationale PME, 10(1), 43-72.

Verstraete, T., \& Fayolle, A. (2005). Paradigmes et entrepreneuriat. Revue de l'Entrepreneuriat, 4(1), 33-52.

Verzat, C. (2014). Engagement, agilité cognitive, coopération et réflexivité des apprenants... et des enseignants en entrepreneuriat. Revue de l'Entrepreneuriat, 13(2), 7-13.

Vignaux, G. (1994). Les sciences cognitives : Une introduction. Paris, FR: Biblio Essais.

Ward, M.K., Reeck, C., \& Becker, W. (2017). A brief primer on using functional magnetic resonance imaging (fMRI) in entrepreneurship research. In M. Day, M.C. Boardman \& N.F. Krueger (Eds.), Handbook of Research Methodologies and Design in Neuroentrepreneurship, pp. 120-149. Cheltenham, UK: Edward Elgar.

Walsh, J.P. 1995. Managerial and organizational cognition: Notes from a trip down memory lane. Organization Science, 6(3): 280-321.

Warnier, V., Lecocq, X., \& Demil, B. (2018). Les business models dans les champs de l'innovation et de l'entrepreneuriat. Discussion et pistes de recherche. Revue de l'Entrepreneuriat, 17(2), 113-131.

Wiklund, J., Hatak, I., Patzelt, H., \& Shepherd, D.A. (2018). Mental disorders in the entrepreneurship context: When being different can be an advantage. Academy of Management Perspectives, 32(2), 182-206.

Wood, M.S.. McKelvie, A. \& Haynie, J.M. (2014). Making it personal: opportunity individuation and the shaping of opportunity beliefs. Journal of Business Venturing, 29(2), 252-272.

Yatribi, T., Balhadj, S., \& Giraud, L. (2017). L'influence de l'environnement de travail sur la satisfaction et l'intention entrepreneuriale des ingénieurs. Revue de l'Entrepreneuriat, 16(3), 45-82.

Zajonc, R.B. (1984). On the primacy of affect. American Psychologist, 39(2), 117-23. 